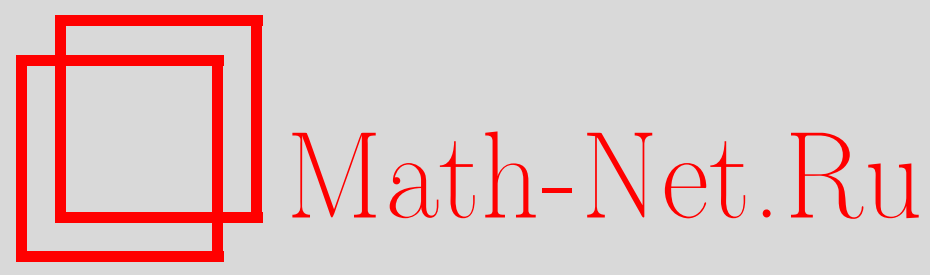

И. А. Бикчантаев, Интегральные операторы И.Н. Векуа на римановой поверхности, Матем. заметки, 2001, том 69, выпуск 1, 18-30

DOI: https://doi.org/10.4213/mzm480

Использование Общероссийского математического портала Math-Net.Ru подразумевает, что вы прочитали и согласны с пользовательским соглашением http://www . mathnet.ru/rus/agreement

Параметры загрузки:

IP : 52.205 .19 .152

26 апреля 2023 г., 15:59:00

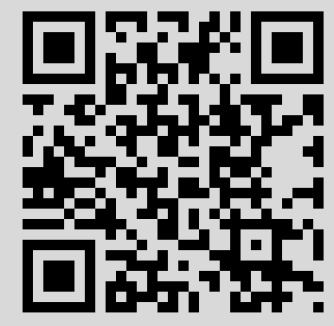




\section{ИНТЕГРАЛЬНЫЕ ОПЕРАТОРЫ И.Н. ВЕКУА НА РИМАНОВОЙ ПОВЕРХНОСТИ}

\section{И. А. Бикчантаев}

На произвольной (вообще говоря, некомпактной) римановой поверхности $R$ исследуются интегральные операторы Т и П, являющиеся аналогами соответствующих операторов, введенных И.Н. Векуа при построении им теории обобщенных аналитических функций. В качестве приложения получены необходимые и достаточные условия разрешшмости неоднородного уравнения Коши-Римана $\bar{\partial} f=F$ в классе функций $f$ с $\Lambda_{0}$-поведением в окрестности идеальной границы поверхности $R$.

Библиограффия: 7 названий.

Неоднородное уравнение Коши-Римана

$$
\bar{\partial} f=F
$$

на компактной римановой поверхности в естественных пространствах является нётеровым, в то время как на некомпактной римановой поверхности $R$ оно разрешимо при любой правой части $F$ и его решение определяется с точностью до произвольной аналитической на $R$ функции [1]. В этой статье уравнение (1) рассматривается на произвольной римановой поверхности в таких классах функций, в которых соответствующее однородное уравнение $(F=0)$ имеет лиш конечное число линейно независимых решений. При построении теории обобщенных аналитических функций на плоскости и, в частности, при исследовании уравнения вида (1) И.Н. Векуа [2] ввел в рассмотрение и изучил интегральные операторы Т и П. Изучению аналогов этих операторов на произвольной (вообще говоря, некомпактной) римановой поверхности в классах квадратично интегрируемых линейных дифференциалов и посвящена в основном эта статья. В качестве приложения получены необходимые и достаточные условия разрешимости уравнения (1) в классе функций с $\Lambda_{0}$-поведением и приведена структурная формула для его решений.

1. Предварительные результаты. В статьях Сиба [3], [4] многие классические результаты теории функций на компактных римановых поверхностях были распространены на случай произвольных некомпактных римановых поверхностей при определенных ограничениях на поведение мероморфных функций и дифференциалов в окрестности идеальной границы (при так назьваемом $\Lambda_{0}$-поведении). В статье [5] было получено необходимое и достаточное условие разрешимости и построено решение уравнения (1) в классе функций с $\Lambda_{0}$-поведением в случае, когда носитель дифференциальной формы $F$ 
компактен. Здесь мы рассмотрим уравнение (1) в случае, когда правая часть есть произвольная линейная квадратично суммируемая дифференциальная форма типа $(0,1)$. При этом данное Сиба [3], [4] определение $\Lambda_{0}$-поведения линейных дифференциалов является недостаточно общим. Поэтому мы начнем с обобщения этого определения на более широкий класс дифференциальных форм.

Пусть $R$ есть произвольная (вообще говоря, некомпактная) риманова поверхность рода $h(\leqslant \infty), J$-множество $\{1,2, \ldots, 2 h\}, \mathscr{R}=\left\{R_{n}\right\}_{n=1}^{\infty}-$ каноническое исчерпание поверхности $R$. Обозначим через $h_{n}$ род поверхности $R_{n}$ и положим $J_{n}=\left\{1,2, \ldots, 2 h_{n}\right\}$. Пусть $\left\{D_{j}\right\}_{j \in J}$ есть канонический базис гомологий поверхности $R$ по модулю разбивающих циклов такой, что $\left\{D_{j}\right\}_{j \in J_{n}}$ есть канонический базис гомологий для $R_{n}$ по модулю границы и $D_{j} \subset R \backslash \bar{R}_{n}$ для $j \in J \backslash J_{n}$. Для удобства положим $R_{0}=\varnothing$ и $J_{0}=\varnothing$.

Пусть $\Lambda=\Lambda(R)$ есть действительное гильбертово пространство квадратично интегрируемых комплексных дифференциалов первого порядка на $R$ с внутренним произведением

$$
\left\langle\lambda_{1}, \lambda_{2}\right\rangle=\operatorname{Re} \iint_{R} \lambda_{1} \wedge * \bar{\lambda}_{2}, \quad \lambda_{1}, \lambda_{2} \in \Lambda .
$$

Норма дифференциала $\lambda \in \Lambda$ определяется равенством $\|\lambda\|=\sqrt{\langle\lambda, \lambda\rangle}$. Положим $\Lambda_{h}=$ $\Lambda_{h}(R)=\{\lambda \in \Lambda: \lambda$ гармонический на $R\}, \Lambda_{\mathrm{hse}}=\Lambda_{\mathrm{hse}}(R)=\left\{\lambda \in \Lambda_{h}: \lambda\right.$ полуточен (т.е. имеет нулевые периоды вдоль всех разбиваюших циклов на $R)\}, \Lambda_{e 0}^{1}=\Lambda_{e 0}^{1}(R)=\{\lambda \in \Lambda$ : существуют $f \in C^{2}(R), f_{n} \in C_{0}^{2}(R)$ такие, что $\mathrm{d} f=\lambda$ и $\left.\left\|\mathrm{d} f-\mathrm{d} f_{n}\right\| \rightarrow 0, n \rightarrow \infty\right\}$, $\Lambda_{c}^{1}=\Lambda_{c}^{1}(R) \quad(\subset \Lambda(R))$ - множество замкнутых дифференциалов класса $C^{1}(R), \Lambda_{c 0}^{1}=$ $\Lambda_{c 0}^{1}(R)$ - подмножество $\Lambda_{c}^{1}$, состоящее из финитных дифференциалов. Через $\Lambda_{e 0}, \Lambda_{c}$, $\Lambda_{c 0}$ обозначим замькания пространств $\Lambda_{e 0}^{1}, \Lambda_{c}^{1}, \Lambda_{c 0}^{1}$ в $\Lambda$. Тогда $\Lambda=\Lambda_{e 0} \oplus \Lambda_{e 0}^{*} \oplus \Lambda_{h}$, где $\Lambda_{e 0}^{*}=\left\{\lambda: * \lambda \in \Lambda_{e 0}\right\}$. Подпространство $\Lambda$, состоящее из действительных дифференциалов, обозначим через $\Gamma$; соответствующие действительные подпространства пространств $\Lambda_{e 0}, \Lambda_{c}, \ldots$ будем обозначать $\Gamma_{e 0}, \Gamma_{c}, \ldots$

Пусть $\mathscr{L}=\left\{L_{j}\right\}_{j=1}^{h}$ семейство прямых в комплексной плоскости $\mathbb{C}$, проходящих через начало координат. Приведем следующее

ОПРЕДЕЛЕНИЕ 1 [4]. Замкнутое подпространство $\Lambda_{0}=\Lambda_{0}(R, \mathscr{L})$ пространства $\Lambda_{\mathrm{hse}}$ назьвается пространством поведения, ассоциированным с $\mathscr{L}$, если $i \Lambda_{0}^{*}=\Lambda_{0}^{\perp}$ (т.е. $\left.\Lambda_{h}=\Lambda_{0} \oplus i \Lambda_{0}^{*}\right)$ и $\int_{D_{j}} \lambda_{0} \in L_{[(j+1) / 2]}, j \in J, \lambda_{0} \in \Lambda_{0}$, где $\Lambda_{0}^{\perp}$ означает ортогональное дополнение $\Lambda_{0}$ в $\Lambda_{h},[\cdot]$ означает целую часть числа.

Пространство $\bar{\Lambda}_{0}=\left\{\lambda: \bar{\lambda} \in \Lambda_{0}\right\}$ является пространством поведения, ассоциированным с семейством прямых $\overline{\mathscr{L}}=\left\{\bar{L}_{j}\right\}_{j=1}^{h}$, где $\bar{L}_{j}$ означает прямую в $\mathbb{C}$, симметричную с $L_{j}$ относительно действительной оси; оно называется двойственным с $\Lambda_{0}[4]$.

Имеет место следующая теорема.

TEOрема 1 (cp. [4]). Пусть $R_{n} \in \mathscr{R}, V=R \backslash \bar{R}_{n} u \varphi \in \Lambda(V)-$ полуточный дифференциал такой, что $\int_{D_{j}} \varphi \in L_{[(j+1) / 2]}, j \in J \backslash J_{n}$. Пусть $\Phi$ есть (вообще говоря, многозначная) функиия на $\bar{V}$ такая, что $\mathrm{d} \Phi=\varphi$. Тогда следуюшие три условия эквивалентны:

(I) существуют $\lambda_{0} \in \Lambda_{0}, \lambda_{e 0} \in \Lambda_{e 0}$ maкие, что $\varphi=\lambda_{0}+\lambda_{e 0}$ на $\mathrm{V}$; 


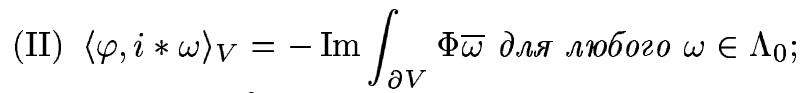

(III) $\lim _{m \rightarrow \infty} \operatorname{Im} \int_{\partial R_{m}} \Phi \bar{\omega}=0$ для любого $\omega \in \Lambda_{0}$.

ДокАЗАТЕльСтво. Эта теорема была доказана Сиба $[4]$ при условии, что $\varphi \in C^{1}(\bar{V})$. Для доказательства того, что (I) $\Longrightarrow(\mathrm{II})$ достаточно заметить, что подмножество дифференциалов класса $C^{1}(\bar{V})$ плотно в $\Lambda(V)$, а функционалы в обеих частях равенства (II) непрерьвны по $\varphi$ в топологии пространства $\Lambda(V)$ (левая часть равенства непрерывна в силу неравенства Шварца, а правая - в силу полуточности $\omega$ и теоремы о следах $[6$, c. 140]). Эквивалентность (II) и (III) следует из легко проверяемого равенства (ср. [4])

$$
\langle\varphi, i * \omega\rangle_{V \cap R_{m}}=-\operatorname{Im} \int_{\partial V} \Phi \bar{\omega}-\operatorname{Im} \int_{\partial R_{m}} \Phi \bar{\omega}, \quad m>n .
$$

Наконец, докажем импликацию (III) $\Longrightarrow(\mathrm{I})$. Доопределим $\varphi$ на всей римановой поверхности $R$ так, чтобы $\varphi \in \Lambda_{c}(R)$. Поскольку дифференциал $\varphi$ полуточен на $V$, то это продолжение можно выбрать точным на $R \backslash \bar{V}=R_{n}$. Пусть $\omega-$ произвольньй дифференциал из $\Lambda_{0}(R)$. Применяя формулу Стокса к области $R_{m}^{\prime}=R_{m} \backslash \bigcup_{\nu=1}^{2 h_{m}} D_{\nu}$ и учитьвая нормировку периодов дифференциалов $\mathrm{d} \Phi$ и $\omega$, имеем

$$
\begin{aligned}
\operatorname{Im} \int_{\partial R_{m}} \Phi \bar{\omega} & =-\langle\mathrm{d} \Phi, \mathrm{i} * \omega\rangle_{R_{m}}-\operatorname{Im} \sum_{\nu=1}^{h_{m}}\left(\int_{D_{2 \nu-1}} \mathrm{~d} \Phi \int_{D_{2 \nu}} \bar{\omega}-\int_{D_{2 \nu}} \mathrm{d} \Phi \int_{D_{2 \nu-1}} \bar{\omega}\right) \\
& =-\langle\mathrm{d} \Phi, \mathrm{i} * \omega\rangle_{R_{m}} .
\end{aligned}
$$

Устремляя в этом равенстве $m$ к бесконечности, из (III) получим, что $\varphi$ ортогонален i $\Lambda_{0}^{*}(R)=\Lambda_{0}^{\perp}(R)$. Теперь из разложения $\Lambda_{c}=\Lambda_{h} \oplus \Lambda_{e 0}$ следует существование дифференциалов $\lambda_{0} \in \Lambda_{0}, \lambda_{e 0} \in \Lambda_{e 0}$ таких, что $\varphi=\lambda_{0}+\lambda_{e 0}$ на $R$. Это завершает доказательство теоремы.

Понятие $\Lambda_{0}$-поведения было определено Сиба [4] для дифференциалов класса $C^{1}(V)$. Обобщая это понятие, мы дадим следующее

ОПРЕДЕЛЕНИЕ 2. Будем говорить, что дифференциал $\varphi$, определенньй в окрестности идеальной гранищы поверхности $R$, имеет $\Lambda_{0}$-поведение, если существуют $\lambda_{0} \in \Lambda_{0}$, $\lambda_{e 0} \in \Lambda_{e 0}$ и $R_{n} \in \mathscr{R}$ такие, что $\varphi=\lambda_{0}+\lambda_{e 0}$ на $R \backslash R_{n}$. Будем говорить, что функция $f$ (вообще говоря, многозначная) имеет $\Lambda_{0}$-поведение, если ее дифференциал $\mathrm{d} f$ имеет $\Lambda_{0}$-поведение.

Теорема 1 характеризует свойства дифференциалов с $\Lambda_{0}$-поведением. Из нее имеем

ПРЕДЛОЖЕНИЕ 1 (ср. [4]). Пусть ч есть полуточныи дифференциал на $\bar{V}=R \backslash R_{n}$, $R_{n} \in \mathscr{R}$, такой, что $\int_{D_{j}} \varphi \in L_{[(j+1) / 2]}, j \in J \backslash J_{n}$. Предположим, что $\varphi$ имеет $\Lambda_{0}$-поведение. Тогда $\varphi$ допускает представление $\varphi=\lambda_{0}+\lambda_{e 0}, \lambda_{0} \in \Lambda_{0}, \lambda_{e 0} \in \Lambda_{e 0}$ на всей $V$. 
ПРЕДЛОЖЕНИЕ 2. Пусть $\mathrm{d} \Phi$ и $\psi$ - дифференииалы с $\Lambda_{0}$-поведением, определенные в некоторой окрестности идеальной граничы поверхности $R$. Тогда

$$
\lim _{m \rightarrow \infty} \operatorname{Im} \int_{\partial R_{m}} \Phi \bar{\psi}=0 \text {. }
$$

ДоКАЗАТЕЛЬСТВО. По условию в некоторой окрестности идеальной границы справедливы представления $\mathrm{d} \Phi=\lambda_{0}+\lambda_{e 0}, \psi=\psi_{0}+\psi_{e 0}$, где $\lambda_{0}, \psi_{0} \in \Lambda_{0}(R), \lambda_{e 0}, \psi_{e 0} \in$ $\Lambda_{e 0}(R)$. С помошью этих равенств доопределим дифференциалы $\mathrm{d} \Phi$ и $\psi$ на всей римановой поверхности $R$. Применяя формулу Стокса к области $R_{m}^{\prime}$ и учитывая нормировку периодов дифференциалов $\mathrm{d} \Phi$ и $\psi$, аналогично тому, как при доказательстве импликации $(\mathrm{III}) \Longrightarrow(\mathrm{I})$ в теореме 1 , получаем равенство

$$
\operatorname{Im} \int_{\partial R_{m}} \Phi \bar{\psi}=-\langle\mathrm{d} \Phi, \mathrm{i} * \psi\rangle_{R_{m}} .
$$

Taк как d $\Phi \in \Lambda_{0} \oplus \Lambda_{e 0}$ и і $* \psi \in \mathrm{i} \Lambda_{0}^{*} \oplus \mathrm{i} \Lambda_{e 0}^{*}=\Lambda_{0}^{\perp} \oplus \Lambda_{e 0}^{*}$, в силу ортогональных разложений $\Lambda=\Lambda_{e 0} \oplus \Lambda_{e 0}^{*} \oplus \Lambda_{h}, \Lambda_{h}=\Lambda_{0} \oplus \mathrm{i} \Lambda_{0}^{*}$ выражение $\langle\mathrm{d} \Phi, \mathrm{i} * \psi\rangle_{R_{m}}$ стремится к нулю при $m \rightarrow \infty$. Предложение 2 доказано.

2. Интегральные операторы Т и П. В статье [3] доказано существование и единственность следуюших нормированных абелевых дифференциалов на $R$, имеющих $\Lambda_{0}$-поведение.

1) Абелевы дифференциалы первого рода $\varphi_{\nu}, \nu=1,2, \ldots, 2 h$, нормированные условиями

$$
\int_{D_{\nu}} \varphi_{\mu}-\gamma_{\mu} D_{\mu} \circ D_{\nu} \in L_{[(\nu+1) / 2]}, \quad \mu, \nu=1,2, \ldots, 2 h,
$$

где $\gamma_{\mu}$ - заданные комплексные числа, не принадлежашие $L_{[(\mu+1) / 2]}, D_{\mu} \circ D_{\nu}$ означает индекс пересечения циклов $D_{\mu}$ и $D_{\nu}$. Дифференциалы $\varphi_{\nu}$ образуют алгебраический базис пространства абелевых дифференциалов первого рода с $\Lambda_{0}$-поведением.

2) Абелевы дифференциалы третьего рода $\omega_{p q}, \sigma_{p q}$, имеющие единственные простые полюсы в точках $p$ и $q$ с вычетами $1, i$ в $p$ и $-1,-i$ в $q$. Периоды дифференциалов $\omega_{p q}$ и $\sigma_{p q}$ удовлетворяют соотношениям

$$
\int_{D_{\nu}} \omega_{p q} \in L_{[(\nu+1) / 2]}, \quad \int_{D_{\nu}} \sigma_{p q} \in L_{[(\nu+1) / 2]}, \quad \nu=1,2, \ldots, 2 h .
$$

Через $\varphi_{\nu}^{\prime}, \omega_{p q}^{\prime}, \sigma_{p q}^{\prime}$ условимся обозначать дифференциалы, которые получаются, если в определении $\varphi_{\nu}, \omega_{p q}, \sigma_{p q} \Lambda_{0}$-поведение заменить на двойственное $\bar{\Lambda}_{0}$-поведение. При этом вместо соотношений (2) будем иметь

$$
\int_{D_{\nu}} \varphi_{\mu}^{\prime}-\gamma_{\mu}^{\prime} D_{\mu} \circ D_{\nu} \in \bar{L}_{[(\nu+1) / 2]}, \quad \mu, \nu=1,2, \ldots, 2 h
$$

где $\gamma_{\mu}^{\prime} \notin \bar{L}_{[(\mu+1) / 2]}$. Для определенности будем считать числа $\gamma_{\mu}$ и $\gamma_{\mu}^{\prime}$ выбранньги таким образом, что $\left|\gamma_{\mu}\right|=1$, i $\gamma_{2 \mu-1}=\mathrm{i} \gamma_{2 \mu} \in L_{\mu}, \gamma_{\mu}^{\prime}=\bar{\gamma}_{\mu}$. 
Положим

$$
H_{1}(p, q)=\frac{1}{2} \mathrm{~d}_{p} \int_{q_{0}}^{q}\left(\omega_{p p_{0}}-i \sigma_{p p_{0}}\right), \quad H_{2}(p, q)=-\frac{1}{2} \mathrm{~d}_{p} \int_{q_{0}}^{q}\left(\omega_{p p_{0}}+i \sigma_{p p_{0}}\right) .
$$

Выражения (3) суть многозначные (по $q$ ) аналоги ядра Коши, соответствуюшие дивизору $1 / q_{0}$, характер многозначности которых описывается равенствами [7]

$$
\int_{D_{\nu}} \mathrm{d}_{q} H_{1}(p, q)=-\pi i \gamma_{\nu} \varphi_{\nu}^{\prime}(p), \quad \int_{D_{\nu}} \mathrm{d}_{q} H_{2}(p, q)=\pi i \gamma_{\nu} \overline{\varphi_{\nu}^{\prime}(p)}, \quad \nu=1,2, \ldots, 2 h .
$$

Через $h_{1}(p, q), h_{2}(p, q)$ обозначим однозначные на $R^{\prime}:=R \backslash \bigcup_{\nu=1}^{2 h} D_{\nu}$ ветви ядер (3), исчезающие при $q=q_{0}$.

Введем следующие банаховы пространства. $L_{\infty}(R)$ - пространство функций на $R$ с конечной нормой $\|f\|=\operatorname{vrai}_{\sup _{q \in R}}|f(q)| ; \quad L_{1}^{(2)}(R)$ - пространство дифференциалов второго порядка, интегрируемых по $R$, с нормой $\|\Psi\|=\iint_{R}|\Psi| ; L_{2}^{(1)}(R)$ - гильбертово пространство дифференциалов первого порядка, интегрируемых с квадратом (в предыдущем пункте оно обозначалось $\Lambda(R)) ; L_{2}^{1,0}(R)$ и $L_{2}^{0,1}(R)$ - подпространства $L_{2}^{(1)}(R)$, состоящие из дифференциалов типа $(1,0)$ и $(0,1)$ соответственно. Очевидно, $L_{2}^{(1)}(R)=L_{2}^{1,0}(R) \oplus L_{2}^{0,1}(R)$. Положим

$$
\begin{gathered}
(\varphi, \psi)_{1}:=\operatorname{Re} \iint_{R} \varphi \wedge \psi, \quad \varphi, \psi \in L_{2}^{(1)}(R) \\
(f, \Psi)_{2}:=\operatorname{Re} \iint_{R} f \Psi, \quad f \in L_{\infty}(R), \quad \Psi \in L_{1}^{(2)}(R) .
\end{gathered}
$$

Билинейная форма $(\cdot, \cdot)_{1}$ связана со скалярным произведением $\langle\cdot, \cdot\rangle$ в гильбертовом пространстве $L_{2}^{(1)}(R)$ соотношением $\langle\varphi, \psi\rangle=(\varphi, * \bar{\psi})_{1}$. Пространства $L_{1}^{(2)}(R)$ и $L_{\infty}(R)$ являются сопряженными относительно билинейной формы $(\cdot, \cdot)_{2}$.

Пусть $U=\left\{q \in R:\left|z(q)-z\left(q_{0}\right)\right|<1\right\}$ - параметрический круг с центром в точке $q_{0} \in R, z(q)$ - локальная униформизирующая. Положим

$$
m_{0}(q)= \begin{cases}\left|z(q)-z\left(q_{0}\right)\right| & \text { при } q \in U, \\ 1 & \text { при } q \in R \backslash U .\end{cases}
$$

Наряду с введенными функциональными пространствами будем рассматривать также пространства с весом

$$
L_{1}^{(2)}\left(R, m_{0}\right):=\left\{\Psi: m_{0} \Psi \in L_{1}^{(2)}(R)\right\} \quad \text { и } L_{2}^{(1)}\left(R, 1 / m_{0}\right):=\left\{\varphi: \varphi / m_{0} \in L_{2}^{(1)}(R)\right\}
$$

с нормами

$$
\|\Psi\|_{L_{1}^{(2)}\left(R, m_{0}\right)}:=\left\|m_{0} \Psi\right\|_{L_{1}^{(2)}(R)}, \quad\|\varphi\|_{L_{2}^{(1)}\left(R, 1 / m_{0}\right)}:=\left\|\varphi / m_{0}\right\|_{L_{2}^{(1)}(R)},
$$

а также соответствующие подпространства $L_{2}^{1,0}\left(R, 1 / m_{0}\right)$ и $L_{2}^{0,1}\left(R, 1 / m_{0}\right)$. 
Введем в пространстве $L_{2}^{(1)}\left(R, 1 / m_{0}\right)$ интегральные операторы

$$
\begin{aligned}
& \mathrm{T} F(q)=\frac{1}{2 \pi i} \iint_{R} h_{1}(p, q) \wedge F(p)+h_{2}(p, q) \wedge \overline{F(p)}, \\
& \widetilde{\mathrm{T}} F(q)=\frac{1}{2 \pi i} \iint_{R} H_{1}(p, q) \wedge F(p)+H_{2}(p, q) \wedge \overline{F(p)} .
\end{aligned}
$$

Очевидно, Т $F$ является однозначной на $R^{\prime}$ ветвью многозначной функции $\widetilde{\mathrm{T}} F$, исчезающей в точке $q_{0}$. Функция $\widetilde{T} F$ удовлетворяет уравнению (1) [5]. Характер многозначности функции Т $F$ определяется формулами

$$
\int_{D_{\nu}} \mathrm{d} \widetilde{\mathrm{T}} F=-i \gamma_{\nu}\left(F, i \varphi_{\nu}^{\prime}\right)_{1}, \quad \nu=1,2, \ldots, 2 h,
$$

вытекающими из формул (4).

Легко видеть, что ker $\mathrm{T}=\operatorname{ker} \widetilde{\mathrm{T}}=L_{2}^{1,0}\left(R, 1 / m_{0}\right)$. Действительно, включение $L_{2}^{1,0}(R$, $\left.1 / m_{0}\right) \subset \operatorname{ker}$ Т очевидно. Обратное включение следует из того, что сужение оператора $\bar{\partial} \widetilde{\mathrm{T}}$ на подпространство $L_{2}^{0,1}\left(R, 1 / m_{0}\right)$ является единичным оператором [5].

В случае, ког да носитель дифференциала $F$ компактен, конечность интеграла Дирихле функции $\widetilde{\mathrm{T}} F$ по некоторой окрестности $V$ идеальной границы легко следовала из возможности дифференцирования и интегрирования в области $V$ выражения для $\widetilde{\mathrm{T}} F$ под знаком интеграла [5]. Для доказательства этого результата в случае произвольного $F$ из $L_{2}^{(1)}(R)$ требуются нетривиальные рассуждения, некоторые идеи которых восходят к Векуа [2]. С этой целью мы введем еще и другие интегральные операторы и исследуем их свойства.

В пространстве $L_{1}^{(2)}\left(R, m_{0}\right)$ рассмотрим интегральньй оператор

$$
\mathrm{T}^{*} \Psi=\frac{\mathrm{i}}{2 \pi} \iint_{R} h_{1}(\cdot, q) \Psi(q)-\overline{h_{2}(\cdot, q) \Psi(q)} .
$$

В случае, когда этот интеграл сходится и $\mathrm{T}^{*} \Psi \in L_{2}^{1,0}\left(R, m_{0}\right)$, справедливы соотношения $\bar{\partial} \mathrm{T}^{*} \Psi=-\Psi$ в $R \backslash\left\{q_{0}\right\}$ и $(\mathrm{T} F, \Psi)_{2}=\left(F, \mathrm{~T}^{*} \Psi\right)_{1}[5]$.

ПРЕДЛОЖЕНИЕ 3. Пусть $\Psi \in L_{1}^{(2)}\left(R, m_{0}\right) u m_{0} \Psi \in C_{0}^{\infty}\left(R^{\prime}\right)$. Тогда

$$
\int_{D_{\nu}} \mathrm{T}^{*} \Psi=\bar{\gamma}_{\nu} \operatorname{Re} \iint_{R} \Psi(q) \int_{q_{0}}^{q} \varphi_{\nu}
$$

әде путь интегрирования в последнем интеграле не пересекает канонических сечений.

ДокАзАТЕЛьСтво. Выражая ядра $h_{i}(p, q)$ через нормированные абелевы дифференциалы третьего рода с $\bar{\Lambda}_{0}$-поведением [7], перепишем выражение $\mathrm{T}^{*} \Psi$ в виде

$$
\mathrm{T}^{*} \Psi=\frac{\mathrm{i}}{2 \pi} \iint_{R} \omega_{q q_{0}}^{\prime} \operatorname{Re} \Psi(q)+\sigma_{q q_{0}}^{\prime} \operatorname{Im} \Psi(q) .
$$

Учитьвая формулы (см. [7])

$$
\int_{D_{\nu}} \omega_{q q_{0}}^{\prime}=-2 \pi \mathrm{i} \bar{\gamma}_{\nu} \operatorname{Re} \int_{q_{0}}^{q} \varphi_{\nu}, \quad \int_{D_{\nu}} \sigma_{q q_{0}}^{\prime}=2 \pi \mathrm{i} \bar{\gamma}_{\nu} \operatorname{Im} \int_{q_{0}}^{q} \varphi_{\nu}
$$

и возможность перестановки интегралов, получим доказьваемое соотношение. 
ПреДЛОЖЕНИЕ 4. Оператор - $\mathrm{T}^{*} \bar{\partial}$ совпадает $\mathrm{c}$ единичным оператором на подмножестве пространства $L_{2}^{1,0}(R)$, состоящем из гладких дифференииалов $c$ компактным в $R^{\prime}$ носителем.

ДокАЗАтЕльСтво. Пусть $\psi \in L_{2}^{1,0}(R)$ и принадлежит классу $C_{0}^{\infty}\left(R^{\prime}\right)$. Тогда с учетом предложения 3 получаем

$$
\begin{aligned}
\int_{D_{\nu}} \mathrm{T}^{*} \bar{\partial} \psi & =\bar{\gamma}_{\nu} \operatorname{Re} \iint_{R}(\bar{\partial} \psi(q)) \int_{q_{0}}^{q} \varphi_{\nu}=\bar{\gamma}_{\nu} \operatorname{Re} \iint_{R^{\prime}} \bar{\partial}\left(\psi(q) \int_{q_{0}}^{q} \varphi_{\nu}\right) \\
& =\bar{\gamma}_{\nu} \operatorname{Re} \iint_{R^{\prime}} \mathrm{d}\left(\psi(q) \int_{q_{0}}^{q} \varphi_{\nu}\right)=\bar{\gamma}_{\nu} \lim _{n \rightarrow \infty} \operatorname{Re} \int_{\partial R_{n}^{\prime}} \psi(q) \int_{q_{0}}^{q} \varphi_{\nu}=0 .
\end{aligned}
$$

Положим $\alpha:=\psi+\mathrm{T}^{*} \bar{\partial} \psi$. Из равенства $\bar{\partial} \mathrm{T}^{*} \Psi=-\Psi$ следует, что дифференциал $\alpha$ голоморфный. Покажем, что он полуточный. Для этого достаточно установить, что при достаточно больших $m \int_{\gamma} \alpha=0$, где $\gamma-$ любая компонента края $\partial R_{m}$. Но при $\operatorname{supp} \psi \subset R_{m}$ это следует из полуточности ядер $H_{k}(p, q)$ по переменной $p$ и возможности переставить порядок интегрирования по $\gamma$ и по $R$. Из предложения 3 следует, что периоды дифференциала $\alpha$ нормированы условием $\int_{D_{j}}$ i $\alpha \in \bar{L}_{[(j+1) / 2]}, j \in J$. При финитных $\Psi$ дифференциал $\mathrm{T}^{*} \Psi$, а стало быть и $\alpha$, имеет і $\bar{\Lambda}_{0}$-поведение [5]. Следовательно (см. [3]), $\alpha=0$. Предложение доказано.

В пространстве $L_{2}^{(1)}\left(R, 1 / m_{0}\right)$ введем интегральньй оператор П := $\partial \widetilde{\mathrm{T}}$.

ПрЕДЛОЖениЕ 5. Оператор $\Pi: L_{2}^{0,1}(R) \rightarrow L_{2}^{1,0}(R)$ сохраняет скалярное произведение, т.е. имеет место тождество $\langle\Pi F, \Pi E\rangle=\langle F, E\rangle, F, E \in L_{2}^{0,1}(R)$.

ДокАЗАТЕльство. Пусть $F$ - дифференциал типа $(0,1)$, причем дифференциал $F / m_{0}$ является гладким и его носитель является компактньм в $R^{\prime}$, и $G \in L_{2}^{1,0}(R)$, причем $\bar{\partial} G$ принадлежит $L_{1}^{(2)}\left(R, m_{0}\right)$ и имеет компактный носитель в $R^{\prime}$. Тогда

$$
\begin{aligned}
\langle\Pi F, G\rangle & =\langle\partial \widetilde{\mathrm{T}} F, G\rangle=\operatorname{Re} \iint_{R} \partial \widetilde{\mathrm{T}} F \wedge * \bar{G} \\
& =\operatorname{Re} \iint_{R} \mathrm{i} \partial \widetilde{\mathrm{T}} F \wedge \bar{G}=\operatorname{Re} \iint_{R^{\prime}} \mathrm{i} \partial \mathrm{T} F \wedge \bar{G} \\
& =-\operatorname{Re}\left(\mathrm{i} \iint_{R} \mathrm{~T} F \partial \bar{G}\right)+\operatorname{Re}\left(\mathrm{i} \int_{\beta \cup \partial R^{\prime}}(\mathrm{T} F) \bar{G}\right) \\
& =-\operatorname{Re} \iint_{R} F \wedge \mathrm{T}^{*}(\mathrm{i} \partial \bar{G})+\operatorname{Re}\left(\mathrm{i} \int_{\beta \cup \partial R^{\prime}} \bar{G} \mathrm{~T} F\right) \\
& =\left\langle F, \mathrm{i} \overline{\mathrm{T}^{*}(\mathrm{i} \partial \bar{G})}\right\rangle+\operatorname{Re}\left(\mathrm{i} \int_{\beta \cup \partial R^{\prime}} \bar{G} \mathrm{~T} F\right) .
\end{aligned}
$$

Здесь $\partial R^{\prime}$ - относительная граница области $R^{\prime}$ на $R$ в топологии, определяемой метрикой Мазуркевича, $\beta$ - идеальная граница поверхности $R$, причем

$$
\int_{\beta} \varphi:=\lim _{n \rightarrow \infty} \int_{\partial R_{n}} \varphi, \quad R_{n} \in \mathscr{R}
$$


Под значением подьнтегрального выражения на $\partial R^{\prime}$ понимается предельное значение изнутри области $R^{\prime}$.

Положим $\Pi^{*} G:=\mathrm{i} \overline{\mathrm{T}^{*}(\mathrm{i} \partial \bar{G})}$. Тогда из (6) получаем

$$
\langle\Pi F, G\rangle=\left\langle F, \Pi^{*} G\right\rangle+\operatorname{Re}\left(\mathrm{i} \int_{\beta \cup \partial R^{\prime}} \bar{G} \mathrm{~T} F\right) .
$$

Подсчитаем второе слагаемое в правой части равенства (7) при $G=\Pi E$, где $E / m_{0}-$ гладкий дифференциал типа $(0,1)$, носитель которого является компактным подмножеством в $R^{\prime}$. Имеем

$$
\begin{gathered}
\operatorname{Re}\left(\mathrm{i} \int_{\beta \cup \partial R^{\prime}} \bar{G} \mathrm{~T} F\right)=\operatorname{Re}\left(\mathrm{i} \int_{\beta \cup \partial R^{\prime}} \overline{\Pi E} \cdot \mathrm{T} F\right)=\operatorname{Re}\left(\mathrm{i} \int_{\beta \cup \partial R^{\prime}} \mathrm{T} F \cdot \overline{\partial \mathrm{T} E}\right) \\
\quad=\operatorname{Re}\left(\mathrm{i} \int_{\beta \cup \partial R^{\prime}}(\bar{\partial}(\mathrm{T} F \cdot \overline{\mathrm{T} E})-F \overline{\mathrm{T} E})\right)=\operatorname{Re}\left(\mathrm{i} \int_{\beta \cup \partial R^{\prime}} \bar{\partial}(\mathrm{T} F \cdot \overline{\mathrm{T} E})\right) .
\end{gathered}
$$

Из равенств (7) и (8) получаем

$$
\langle\Pi F, \Pi E\rangle=\left\langle F, \Pi^{*} \Pi E\right\rangle+\operatorname{Re}\left(\mathrm{i} \int_{\beta \cup \partial R^{\prime}} \bar{\partial}(\mathrm{T} F \cdot \overline{\mathrm{T} E})\right) .
$$

Покажем, что последнее слагаемое в (9) равно нулю. Действительно, учитывая формулы (5), имеем

$$
\begin{aligned}
\int_{\partial R^{\prime}} \bar{\partial}(\mathrm{T} F \cdot \overline{\mathrm{T} E})= & \int_{\partial R^{\prime}} \mathrm{T} F \cdot \overline{\partial \mathrm{T} E}=\int_{\partial R^{\prime}} \mathrm{T} F \cdot \mathrm{d} \overline{\mathrm{T} E} \\
= & \sum_{\nu=1}^{h}\left(\int_{D_{2 \nu-1}}\left(-\int_{D_{2 \nu}} \mathrm{d} \widetilde{\mathrm{T}} F\right) \mathrm{d} \overline{\widetilde{\mathrm{T}} E}+\int_{D_{2 \nu}}\left(\int_{D_{2 \nu-1}} \mathrm{~d} \widetilde{\mathrm{T}} F\right) \mathrm{d} \overline{\widetilde{\mathrm{T}} E}\right) \\
= & \sum_{\nu=1}^{h}\left(\int_{D_{2 \nu-1}} \mathrm{~d} \widetilde{\mathrm{T}} F \int_{D_{2 \nu}} \mathrm{d} \overline{\widetilde{\mathrm{T}} E}-\int_{D_{2 \nu}} \mathrm{d} \widetilde{\mathrm{T}} F \int_{D_{2 \nu-1}} \mathrm{~d} \overline{\widetilde{\mathrm{T}} E}\right) \\
= & \sum_{\nu=1}^{h}\left(\left(-\mathrm{i} \gamma_{2 \nu-1} \operatorname{Im} \iint_{R} \varphi_{2 \nu-1}^{\prime} \wedge F\right)\left(\mathrm{i} \bar{\gamma}_{2 \nu} \operatorname{Im} \iint_{R} \varphi_{2 \nu}^{\prime} \wedge E\right)\right. \\
& \left.-\left(-\mathrm{i} \gamma_{2 \nu} \operatorname{Im} \iint_{R} \varphi_{2 \nu}^{\prime} \wedge F\right)\left(\mathrm{i} \bar{\gamma}_{2 \nu-1} \operatorname{Im} \iint_{R} \varphi_{2 \nu-1}^{\prime} \wedge E\right)\right) \\
= & \sum_{\nu=1}^{h}\left(\operatorname{Im} \iint_{R} \varphi_{2 \nu-1}^{\prime} \wedge F \cdot \operatorname{Im} \iint_{R} \varphi_{2 \nu}^{\prime} \wedge E\right. \\
& \left.-\operatorname{Im} \iint_{R} \varphi_{2 \nu}^{\prime} \wedge F \cdot \operatorname{Im} \iint_{R} \varphi_{2 \nu-1}^{\prime} \wedge E\right) .
\end{aligned}
$$

Правая часть формулы (10) вешественна, откуда следует, что

$$
\operatorname{Re}\left(\mathrm{i} \int_{\partial R^{\prime}} \bar{\partial}(\overline{\mathrm{T} F} \cdot \mathrm{T} E)\right)=0
$$


Так как носители дифференциалов $F$ и $E$ компактны, то дифференциалы $\mathrm{d} \widetilde{\mathrm{T}} F$ и $\mathrm{d} \widetilde{\mathrm{T}} E$ имеют $\Lambda_{0}$-поведение [5]. Поэтому из предложения 2 следует, что интеграл

$$
\int_{\beta} \bar{\partial}(\mathrm{T} F \cdot \overline{\mathrm{T} E})=\int_{\beta} \mathrm{T} F \cdot \mathrm{d} \overline{\mathrm{T} E}=\int_{\beta} \widetilde{\mathrm{T}} F \cdot \mathrm{d} \overline{\widetilde{\mathrm{T}} E}
$$

вещественньй. Таким образом, из равенства (9) получаем

$$
\langle\Pi F, \Pi E\rangle=\left\langle F, \Pi^{*} \Pi E\right\rangle .
$$

Из определения операторов П и П*, равенства $\bar{\partial} \widetilde{T}=$ I и предложения 4 имеем

$$
\Pi^{*} \Pi E=\mathrm{i} \overline{\mathrm{T}^{*}(\mathrm{i} \partial \overline{\partial \widetilde{\mathrm{T}} E)}}=\mathrm{i} \overline{\mathrm{T}^{*}(\mathrm{i} \overline{\partial E})}=\mathrm{i} \overline{(\mathrm{i} \bar{E})}=E .
$$

Поскольку это равенство имеет место на плотном в $L_{2}^{0,1}(R)$ подмножестве, то $\Pi^{*} \Pi=\mathrm{I}-$ единичньй оператор в пространстве $L_{2}^{0,1}(R)$. Отсюда и из равенства $(11)$ следует, что

$$
\langle\Pi F, \Pi E\rangle=\langle F, E\rangle \text {. }
$$

Так как равенство (12) установлено для плотного в $L_{2}^{0,1}(R)$ подмножества и билинейная форма $\langle\cdot, \cdot\rangle$ непрерьвна в $L_{2}^{(1)}(R)$, то предложение 5 доказано.

СлЕДСТВИЕ 1. Onератор $\Pi: L_{2}^{0,1}(R) \rightarrow L_{2}^{1,0}(R)$ изометричен $u$, стало быть, его норма равна единиие. Oператор $\Pi^{*}: L_{2}^{1,0}(R) \rightarrow L_{2}^{0,1}(R)$ является для него левым.м обратным.

ПРЕДЛОЖЕНИЕ 6. Функиия $\widetilde{\mathrm{T}} F, F \in L_{2}^{(1)}(R)$, имеет $\Lambda_{0}$-поведение.

ДокАЗАтЕЛЬСтво. Из следствия 1 вытекает, что Т $F$ имеет конечный интеграл Дирихле. Так как ядра $H_{i}(p, q)$ по переменной $q$ не имеют периодов вдоль разбивающих циклов, то дифференциал $\mathrm{d} \widetilde{\mathrm{T}} F$ полуточен на $R$. Из формул (5) следует, что периоды $\mathrm{d} \widetilde{\mathrm{T}} F$ вдоль канонических циклов нормированы. Для завершения доказательства того, что $\widetilde{\mathrm{T}} F$ имеет $\Lambda_{0}$-поведение достаточно согласно теореме 1 установить соотношение

$$
\langle\mathrm{d} \widetilde{T} F, \mathrm{i} * \omega\rangle=0 \quad \text { для всех } \omega \in \Lambda_{0} .
$$

Для финитных в $R$ дифференциалов $F$ функция $\widetilde{T} F$ имеет $\Lambda_{0}$-поведение [5], откуда и следует соотношение $(13)$. Так как $\mathrm{d} \widetilde{T} F=(\mathrm{I}+\Pi) F$, оператор $\mathrm{I}+\Pi$ и билинейная форма $\langle\cdot, \cdot\rangle$ непрерывны в пространстве $L_{2}^{(1)}(R)$, то $(13)$ верно для всех $F \in L_{2}^{(1)}(R)$. Предложение 6 доказано.

ПРЕДЛОЖЕНИЕ 7. Оператор $\mathrm{d} \widetilde{\mathrm{T}}$ ограничен в пространстве $L_{2}^{(1)}(R)$ и его ядром служит подпространство $L_{2}^{1,0}(R)$. Подпространство $L_{2}^{0,1}(R)$ он взаимно однозначно отображсает на подпространство $\Lambda_{0}(R) \oplus \Lambda_{e 0}(R)$. 
ДокАЗАтЕЛЬСтво. Включение $L_{2}^{1,0}(R) \subset \operatorname{ker} \mathrm{d} \widetilde{\mathrm{T}}$ очевидно. Поэтому ограниченность оператора $\mathrm{d} \widetilde{\mathrm{T}}$ вытекает из представления $L_{2}^{(1)}(R)=L_{2}^{1,0}(R) \oplus L_{2}^{0,1}(R)$, равенства $\mathrm{d} \widetilde{\mathrm{T}}=\mathrm{I}+\Pi$ на $L_{2}^{0,1}(R)$ и следствия 1 . Из свойств оператора $\widetilde{\mathrm{T}}$, теоремы 1 и предложения 1 вытекает, что образ $\mathrm{im} \mathrm{d} \widetilde{\mathrm{T}}$ оператора $\mathrm{d} \widetilde{\mathrm{T}}$ принадлежит подпространству $\Lambda_{0}(R) \oplus \Lambda_{e 0}(R)$

Рассмотрим уравнение

$$
\mathrm{d} \widetilde{\mathrm{T}} \psi=\varphi, \quad \psi \in L_{2}^{0,1}(R), \quad \varphi \in \Lambda_{0}(R) \oplus \Lambda_{e 0}(R)
$$

Оно эквивалентно уравнению $(\widetilde{\mathrm{T}} \psi)(q)=\int_{q_{0}}^{q} \varphi$. (Равенство этих двух многозначных функций понимается в том смысле, что на $R^{\prime}$ совпадают их однозначные ветви, исчезающие при $q=q_{0}$, а также ветви, которые получаются из отмеченных непрерьвным продолжением вдоль одного и того же пути.) Применяя оператор $\bar{\partial}$ к обеим частям последнего равенства, получаем $\psi(q)=\bar{\partial} \int_{q_{0}}^{q} \varphi$. Отсюда, в частности, вытекает, что оператор $\mathrm{d} \widetilde{\mathrm{T}}$ однозначно разрешим в подпространстве $L_{2}^{0,1}(R)$ и, следовательно, ker $\mathrm{d} \widetilde{\mathrm{T}}=L_{2}^{1,0}(R)$.

Для доказательства везде разрешимости оператора $\mathrm{d} \widetilde{\mathrm{T}}: L_{2}^{0,1}(R) \rightarrow \Lambda_{0}(R) \oplus \Lambda_{e 0}(R)$ достаточно показать, что любой дифференциал вида $\psi=\bar{\partial} \int_{q_{0}}^{q} \varphi$, где $\varphi \in \Lambda_{0} \oplus \Lambda_{e 0}$, удовлетворяет уравнению $(\widetilde{\mathrm{T}} \psi)(q)=\int_{q_{0}}^{q} \varphi$, т.е. $\widetilde{\mathrm{T}} \bar{\partial} \int_{q_{0}}^{q} \varphi=\int_{q_{0}}^{q} \varphi$. Легко видеть, что функция $\widetilde{\mathrm{T}} \bar{\partial} \int_{q_{0}}^{q} \varphi-\int_{q_{0}}^{q} \varphi$ (под суммой этих двух многозначных функций имеется в виду функция, одна из однозначных ветвей которой на $R^{\prime}$ равна сумме однозначных ветвей этих функций, исчезающих в точке $q_{0}$; остальные ветви получаются продолжением слагаемых вдоль одного и того же пути) аналитична на $R$, имеет $\Lambda_{0}$-поведение, ее периоды вдоль разбивающих циклов равны нулю, а периоды вдоль канонических циклов нормированы и одна из ее однозначных ветвей исчезает в точке $q_{0}$. По теореме единственности [3] она равна нулю. Предложение 7 доказано.

ПРЕДЛОЖЕНИЕ 8. Имеем

$$
\widetilde{\mathrm{T}} \bar{\partial} f-f=\mathrm{const}
$$

для многозначных на $R$ функиий $f$ maких, что $\mathrm{d} f \in \Lambda_{0}(R) \oplus \Lambda_{e 0}(R)$.

ДокАЗАтЕльство. Положим $\widetilde{\mathrm{T}} \bar{\partial} f=g$. Тогда $\bar{\partial}(f-g)=0$. Таким образом, $f-g$ есть аналитическая на $R$ функция с $\Lambda_{0}$-поведением. Следовательно, $f-g$ постоянна [3], что и требовалось доказать.

ПРЕДЛОЖЕНИЕ 9. Onератор $\Pi^{*}: L_{2}^{(1,0)}(R) \rightarrow L_{2}^{(0,1)}(R)$ является обратнылм для onepamopa $\Pi: L_{2}^{0,1}(R) \rightarrow L_{2}^{1,0}(R)$.

ДокАЗАТЕЛЬСтво. Введем следующие обозначения:

$$
H_{1}^{\prime}(q, p):=\frac{1}{2} \mathrm{~d}_{q} \int_{p_{0}}^{p}\left(\omega_{q q_{0}}^{\prime}-\mathrm{i} \sigma_{q q_{0}}^{\prime}\right), \quad H_{2}^{\prime}(q, p):=-\frac{1}{2} \mathrm{~d}_{q} \int_{p_{0}}^{p}\left(\omega_{q q_{0}}^{\prime}+\mathrm{i} \sigma_{q q_{0}}^{\prime}\right) .
$$

Ядра $H_{k}^{\prime}(q, p), k=1,2$, отличаются от ядер $H_{k}(p, q)$ заменой $p$ и $p_{0}$ соответственно на $q$ и $q_{0}$ и наоборот, а также заменой $\Lambda_{0}$-поведения на двойственное $\bar{\Lambda}_{0}$-поведение. Положим

$$
\widetilde{\mathrm{T}}^{\prime} F(p):=\frac{1}{2 \pi \mathrm{i}} \iint_{R} H_{1}^{\prime}(q, p) \wedge F(q)+H_{2}^{\prime}(q, p) \wedge \overline{F(q)}
$$


и через $\mathrm{T}^{\prime} F$ обозначим однозначную на $R^{\prime}$ ветвь этой функции, исчезающую в точке $p_{0}$. Положим $\Pi^{\prime}=\partial \widetilde{\mathrm{T}}^{\prime}$ и $\bar{\Pi}^{\prime} G:=\overline{\Pi^{\prime} \bar{G}}$.

Установим равенство

$$
\Pi^{*}=\bar{\Pi}^{\prime}
$$

Используя формулы перестановки аргумента с параметром в нормированных абелевых дифференциалах третьего рода [7], представим ядра $H_{k}(p, q)$ в виде

$$
H_{1}(p, q)=\frac{1}{2}\left(\omega_{q q_{0}}^{\prime}(p)-\mathrm{i} \sigma_{q q_{0}}^{\prime}(p)\right), \quad H_{2}(p, q)=-\frac{1}{2} \overline{\left(\omega_{q q_{0}}^{\prime}(p)+\mathrm{i} \sigma_{q q_{0}}^{\prime}(p)\right)} .
$$

Преобразуем выражение $\mathrm{T}^{*}(\mathrm{i} \partial \bar{G})$, считая $G$ гладким дифференциалом типа $(1,0)$ с носителем в $R^{\prime}$. Обозначим через $z$ и $\zeta$ локальные параметры точек $q$ и $p$ соответственно и через $d_{\varepsilon}-$ параметрический круг на $R$ с центром в точке $p$, определяемый неравенством $|z-\zeta|<\varepsilon$. Тогда

$$
\begin{aligned}
\mathrm{T}^{*}(\mathrm{i} \partial \bar{G})(p)= & \frac{\mathrm{i}}{2 \pi} \iint_{R} H_{1}(p, q) \mathrm{i} \partial \overline{G(q)}-\overline{H_{2}(p, q) \mathrm{i} \partial \overline{G(q)}} \\
= & \frac{\mathrm{i}}{2 \pi}\left(\iint_{R \backslash d_{\varepsilon}}+\iint_{d_{\varepsilon}}\right)\left(\frac{1}{2}\left(\omega_{q q_{0}}^{\prime}(p)-\mathrm{i} \sigma_{q q_{0}}^{\prime}(p)\right) \mathrm{i} \partial \overline{G(q)}\right. \\
& \left.+\frac{1}{2}\left(\omega_{q q_{0}}^{\prime}(p)+\mathrm{i} \sigma_{q q_{0}}^{\prime}(p)\right) \overline{\mathrm{i} \partial \overline{G(q)}}\right) .
\end{aligned}
$$

Интеграл по $d_{\varepsilon}$ в правой части этого равенства стремится к нулю при $\varepsilon \rightarrow 0$. Интеграл по $R \backslash d_{\varepsilon}$ преобразуем интегрированием по частям

$$
\begin{aligned}
& \frac{\mathrm{i}}{2 \pi} \iint_{R \backslash d_{\varepsilon}}\left(\frac{1}{2}\left(\omega_{q q_{0}}^{\prime}(p)-\mathrm{i} \sigma_{q q_{0}}^{\prime}(p)\right) \mathrm{i} \partial \overline{G(q)}+\frac{1}{2}\left(\omega_{q q_{0}}^{\prime}(p)+\mathrm{i} \sigma_{q q_{0}}^{\prime}(p)\right) \overline{\mathrm{i} \partial \overline{G(q)}}\right) \\
& \quad=\frac{1}{2 \pi} \iint_{R \backslash d_{\varepsilon}}\left(\frac{1}{2} \mathrm{~d}_{q}\left(\omega_{q q_{0}}^{\prime}(p)-\mathrm{i} \sigma_{q q_{0}}^{\prime}(p)\right) \wedge \overline{G(q)}-\frac{1}{2} \mathrm{~d}_{q}\left(\omega_{q q_{0}}^{\prime}(p)+\mathrm{i} \sigma_{q q_{0}}^{\prime}(p)\right) \wedge G(q)\right) \\
& \quad-\frac{1}{2 \pi} \int_{\partial d_{\varepsilon}}\left(\frac{1}{2}\left(\omega_{q q_{0}}^{\prime}(p)-\mathrm{i} \sigma_{q q_{0}}^{\prime}(p)\right) \overline{G(q)}-\frac{1}{2}\left(\omega_{q q_{0}}^{\prime}(p)+\mathrm{i} \sigma_{q q_{0}}^{\prime}(p)\right) G(q)\right) .
\end{aligned}
$$

Первое слагаемое в правой части этого равенства перепишем в виде

$$
\frac{1}{2 \pi} \partial \iint_{R \backslash d_{\varepsilon}}\left(H_{1}^{\prime}(q, p) \wedge \overline{G(q)}+H_{2}^{\prime}(q, p) \wedge G(q)\right) .
$$

Вычислим предел интеграла по $\partial d_{\varepsilon}$ в равенстве $(16)$ при $\varepsilon \rightarrow 0$. Так как $\omega_{q q_{0}}^{\prime}$ и $\sigma_{q q_{0}}^{\prime}$ имеют в точке $q$ полюс первого порядка с вычетом 1 и і соответственно, то дифференциал $\omega_{q q_{0}}^{\prime}+\mathrm{i} \sigma_{q q_{0}}^{\prime}$ в точке $q$ регулярен и поэтому предел интеграла по $\partial d_{\varepsilon}$ от $\left(\omega_{q q_{0}}^{\prime}+\mathrm{i} \sigma_{q q_{0}}^{\prime}\right) G(q)$ равен нулю. Полагая $G(q)=g(z) \mathrm{d} z$, интеграл по $\partial d_{\varepsilon}$ от первого подьнтегрального слагаемого запишем в виде

$$
\begin{aligned}
& \int_{\partial d_{\varepsilon}} \frac{1}{2}\left(\omega_{q q_{0}}^{\prime}-\mathrm{i} \sigma_{q q_{0}}^{\prime}\right) \overline{G(q)}=\mathrm{d} \zeta \int_{|z-\zeta|=\varepsilon}\left(\frac{1}{\zeta-z}+a(\zeta, z)\right) \overline{g(z) \mathrm{d} z} \\
& \quad=\mathrm{d} \zeta \int_{|z-\zeta|=\varepsilon} a(\zeta, z) \overline{g(z) \mathrm{d} z}+\mathrm{d} \zeta \int_{|z-\zeta|=\varepsilon} \frac{(\overline{g(z)-g(\zeta)}) \overline{\mathrm{d} z}}{\zeta-z}+\overline{g(\zeta)} \mathrm{d} \zeta \int_{|z-\zeta|=\varepsilon} \frac{\mathrm{d} \bar{z}}{\zeta-z}
\end{aligned}
$$


где функция $a(\zeta, z)$ голоморфная по $\zeta$ и $z$. Последний интеграл в этом равенстве равен нулю, а два первых интеграла справа стремятся к нулю при $\varepsilon \rightarrow 0$. Теперь очевидно, что предел интеграла по $\partial d_{\varepsilon}$ в равенстве $(16)$ при $\varepsilon \rightarrow 0$ равен нулю. Переходя к пределу при $\varepsilon \rightarrow 0$ в равенстве (15) и учитывая $(16),(17)$, получим формулу

$$
\mathrm{T}^{*}(\mathrm{i} \partial \bar{G})=\mathrm{i} \partial \widetilde{\mathrm{T}}^{\prime} \bar{G}
$$

из которой вытекает формула (14).

Из предложения 7 , примененного к оператору $\mathrm{d} \widetilde{\mathrm{T}}^{\prime}$, вытекает, что im $\mathrm{d} \widetilde{\mathrm{T}}^{\prime}=\overline{\Lambda_{0}(R)} \oplus$ $\Lambda_{e 0}(R)$ и, значит, im d $\widetilde{\mathrm{T}}^{\prime}=\Lambda_{0}(R) \oplus \Lambda_{e 0}(R)$. Используя формулу $(14)$, предложения 6

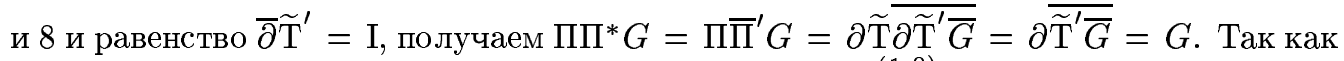
соотношение $\Pi \Pi^{*} G=G$ установлено для плотного в $L_{2}^{(1,0)}(R)$ подмножества дифференциалов $G$, то предложение 9 доказано.

СлЕДСТВИЕ 2. Onератор $\Pi^{*}: L_{2}^{(1,0)}(R) \rightarrow L_{2}^{(0,1)}(R)$ изометрический и его норма равна единице.

СлЕДСТВИЕ 3. Onератор $\Pi^{*}: L_{2}^{(1,0)}(R) \rightarrow L_{2}^{(0,1)}(R)$ является сопряженным onepamopy $\Pi: L_{2}^{0,1}(R) \rightarrow L_{2}^{1,0}(R)$.

ДокАЗАТЕЛЬСтво. Из равенства (7) для гладких и финитных в $R^{\prime}$ дифференциалов $F$ и $G$ получаем $\langle\Pi F, G\rangle=\left\langle F, \Pi^{*} G\right\rangle$. Так как множество таких дифференциалов плотно в $L_{2}^{(0,1)}(R)$ и $L_{2}^{(1,0)}(R)$ соответственно, а операторы П и П* непрерьвны, то это равенство справедливо при всех $F \in L_{2}^{(0,1)}(R)$ и $G \in L_{2}^{(1,0)}(R)$, что и требовалось доказать.

Отметим теперь случай, когда все прямые $L_{j}$, образующие $\mathscr{L}$, суть мнимые оси і $\mathbb{R}$ и $\bar{\Lambda}_{0}=\Lambda_{0}$. Примером такого пространства поведения служит пространство $\Lambda_{K}:=$ $\Gamma_{h m}+\mathrm{i} \Gamma_{\text {hse }}$, где $\Gamma_{h m}$ есть ортогональное дополнение для $\Gamma_{\text {hse }}^{*}$ в $\Gamma_{h}$. Мероморфные функции и дифференциалы с $\Lambda_{K}$-поведением были введены (в иных терминах) и изучены независимо друг от друга Кусуноки, Альфорсом и Ройденом. При таком выборе пространства поведения $\Pi^{\prime}=\Pi, \Pi^{*}=\bar{\Pi}$.

В случае, когда риманова поверхность $R$ является комплексной плоскостью, в силу наличия глобальной системы координат операторы Т и П обычно рассматривают в пространстве функций, а не дифференциальных форм. Если при этом в качестве точки $q_{0}$ выбрать бесконечно удаленную точку, то построенные здесь операторы Т и П совпадут с соответствующими операторами Векуа [2].

\section{3. Решение уравнения (1).}

ПРЕДЛОЖЕНИЕ 10. Пусть $F \in L_{2}^{(0,1)}(R)$. Для существования регулярного (регулярность решения $f$ означает, что $\left.\mathrm{d} f \in L_{2}^{(1)}(R)\right)$ на $R$ решения уравнения (1) в классе функиий с $\Lambda_{0}$-поведением необходимо и достаточно выполнения условий

$$
\operatorname{Im} \iint_{R} F \wedge \varphi_{\nu}^{\prime}=0, \quad \nu=1,2, \ldots, 2 h,
$$

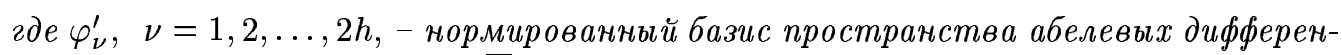
ииалов первого рода на $R$ с $\bar{\Lambda}_{0}$-поведением. При их выполнении общее решение имеет вид

$$
f=\widetilde{\mathrm{T}} F+c=\mathrm{T} F+c
$$


где с - произвольная комплексная постоянная.

ДокАЗАТЕЛЬСТво. Необходимость условий (18) следует из равенств

$$
\operatorname{Im} \iint_{R} F \wedge \varphi_{\nu}^{\prime}=-\left(F, i \varphi_{\nu}^{\prime}\right)_{1}=-\left(\bar{\partial} f, \mathrm{i} \varphi_{\nu}^{\prime}\right)_{1}=\left(f, \mathrm{i} \bar{\partial} \varphi_{\nu}^{\prime}\right)_{2}=0, \quad \nu=1,2, \ldots, 2 h .
$$

Достаточность. Из следствия 1 и предложения 6 вытекает, что $\mathrm{d} f \in L_{2}^{(1)}(R)$ и $f$ имеет $\Lambda_{0}$-поведение независимо от условий (18). Учитывая соотношения (5), мы видим, что условия (18) обеспечивают однозначность функции $\widetilde{\mathrm{T}} F$. Из равенства $\bar{\partial} \widetilde{\mathrm{T}} F=F$ следует, что $f$ удовлетворяет уравнению (1).

Предложение доказано.

\section{СПИСОК ЦИТИРОВАННОЙ ЛИТЕРАТУРЫ}

[1] Форстер О. Римановы поверхности. М.: Мир, 1980.

[2] Векуа И.Н. Обобщенные аналитические функции. М.: Наука, 1988.

[3] Shiba M. On the Riemann-Roch theorem on open Riemann surfaces // J. Math. Kyoto Univ. 1971. V. 11. №3. P. $495-525$.

[4] Shiba M. Some general properties of behavior spaces of harmonic semiexact differentials on an open Riemann surface // Hiroshima Math. J. 1978. V. 8. № 1. P. 151-164.

[5] Бикчантаев И.А. Интегральные представления решений эллиптических систем первого порядка на римановых поверхностях // Дифференц. уравнения. 1986. Т. 22. № 9. С. 1557-1565.

[6] Михайлов В. П. Дифференциальные уравнения в частных производных. М.: Наука, 1976.

[7] Бикчантаев И. А. Аналоги ядра Коши на римановой поверхности и некоторые их приложения // Матем. сб. 1980. Т. 112. № 2. С. 256-282. 